\title{
PERIOD BOUND FOR AUTONOMOUS LIÉNARD OSCILLATIONS*
}

By R. A. SMITH (University of Durham, Durham, England)

1. Introduction. This paper concerns the generalized Liénard differential equation

$$
x^{\prime \prime}-k x^{\prime} f(x)+g(x)=0
$$

where prime denotes differentitation with respect to the real variable $t, k$ is a positive parameter and $f(x), g(x)$ are continuous real functions of the real variable $x$. Also, $g(x)$ satisfies a Lipschitz condition or some other condition which ensures uniqueness of solution of the initial value problem. Furthermore,

$$
\operatorname{sign} g(x)=\operatorname{sign} x,
$$

for all $x$, where sign $y=1,0,-1$ for $y>0, y=0, y<0$, respectively. Sufficient conditions for (1) to have a nonconstant periodic solution have been given by various authors [6, pp. 305, 321]. It is assumed throughout this paper that (1) has at least one periodic solution $x(t)$ with least period $T$. When $k$ is small, an expression for $T$ can be obtained by Poincare's method [1, p. 112]. Also, Liénard and later writers have given asymptotic formulae for $T$ as $k \rightarrow+\infty$ (see [1, Chapters 4, 6] and [5]). But for values of $k$ between these extremes little seems to be known about $T$. For the special case of van der Pol's equation (i.e. when $f(x)=1-x^{2}, g(x)=x$ ), $T$ has been computed numerically in the range $0.1 \leq k \leq 20$ by Urabe [7, p. 222] and others. Also, Graffi [3] showed that if there exists a constant $H$ such that $x g(x) \leq H x^{2}$ for all $x$ then two successive zeros $t_{1}, t_{2}$ of any solution $x(t)$ of (1) satisfy $\left|t_{1}-t_{2}\right| \geq \pi / \sqrt{ } H$. This gives $2 \pi / \sqrt{ } H$ as a lower bound for $T$ since any periodic solution has at least three zeros in some closed interval of length $T$ because of (2). The object of the present paper is to provide a simple upper bound for $T$ which is valid for all $k$. Write

$$
F(x)=\int_{0}^{x} f(\xi) d \xi, \quad G(x)=\int_{0}^{x} g(\xi) d \xi
$$

and assume that positive constants $v, a, \alpha, b, \beta$ exist such that

$$
\begin{aligned}
2 v^{2} G(x) & \leq g(x)^{2} \text { for }-a<x<\alpha, \\
f(x) & \neq 0 \text { for }-b<x<\beta .
\end{aligned}
$$

If $g(x)$ is differentiable then (2) and the equation

$$
2 v^{2} G(x)-g(x)^{2}=2 \int_{0}^{x}\left(v^{2}-d g / d \xi\right) g(\xi) d \xi
$$

show that $v^{2} \leq d g / d x$ in $(-a, \alpha)$ is a sufficient condition for (4).

THEOREM 1. Suppose that $f(x), g(x)$ satisfy (2), (4), (5). If $x(t)$ is a periodic solution of (1) with least period $T$ which has $-a \leq x(t) \leq \alpha$ for all $t$ then

$$
T \leq v^{-1}\left\{8+6 \gamma^{-1 / 2}\left(J_{1}+J_{2}\right)+8 \gamma^{-1 / 2} \max \left(J_{1}, J_{2}\right)\right\},
$$

\footnotetext{
* Received December 9, 1968.
} 
where

$$
J_{1}=k \int_{0}^{\alpha} f_{+}(x) d x, \quad J_{2}=k \int_{-a}^{0} f_{+}(x) d x, \quad f_{+}(x)=\max (0, f(x))
$$

and $\gamma=\min \{2 G(-b), 2 G(\beta)\}$.

In practice, it is often easier to prove the existence of a periodic solution $x(t)$ than to determine constants $a, \alpha$ satisfying $-a \leq x(t) \leq \alpha$. But in cases when (4) holds in $-\infty<x<\infty$ and $\int_{-\infty}^{+\infty} f_{+}(x) d x$ converges, this task can be avoided by taking $a, \alpha=+\infty$ in (6). When this is done for van der Pol's equation, (6) gives the estimate $T \leq 8+$ $(40 k / 3)$ which, for small $k$, is close to the result $T=2 \pi+O\left(k^{2}\right)$ obtained from the Poincaré expansion [1, p. 117]. But for large $k$ it is a cruder estimate of the asymptotic formula $T \sim 1.614 k$ of Liénard [1, p. 105]. This discrepancy is to be expected because very little has been assumed about $f(x)$ in Theorem 1 . The inequality (6) is sharpened in Secs. 3 and 4 at the cost of more restrictive hypotheses on $f(x)$.

2. Proof of Theorem 1. From (3), $G(0)=0$ and from (2), $G(x)$ is strictly decreasing in $(-\infty, 0)$ and strictly increasing in $(0, \infty)$. The continuous function

$$
\phi(x)=[2 G(x)]^{1 / 2} \operatorname{sign} x
$$

is therefore strictly increasing in $-\infty<x<\infty$. Also $\phi(x)$ is differentiable in this range, except possibly at $x=0$. If $y(t)=x^{\prime}(t)-k F(x(t))$, where $x(t)$ is a periodic solution of (1) and $F(x)$ is given by (3), then $x(t), y(t)$ is a periodic solution of the Liénard system

$$
x^{\prime}=y+k F(x), \quad y^{\prime}=-g(x)
$$

and as $t$ varies the point $(x(t), y(t))$ describes a simple closed curve $\Gamma$ in the $(x, y)$ plane. From (2) and (8),

$$
\operatorname{sign} x^{\prime}=\operatorname{sign}[y+k F(x)], \quad \operatorname{sign} y^{\prime}=-\operatorname{sign} x .
$$

The trajectories of (8) therefore cross the curves $y=-k F(x)$ and $x=0$ in the directions shown by the arrows in Figure 1 and $\Gamma$ must encircle the origin in the clockwise sense as $t$ increases. Also (9) shows that $x^{\prime}$ and $y^{\prime}$ are of constant sign along each of the four $\operatorname{arcs} P Q, Q R, R S, S P$ of $\Gamma$. Therefore $y$ and $\phi(x)$ are monotonic functions of $t$ along each

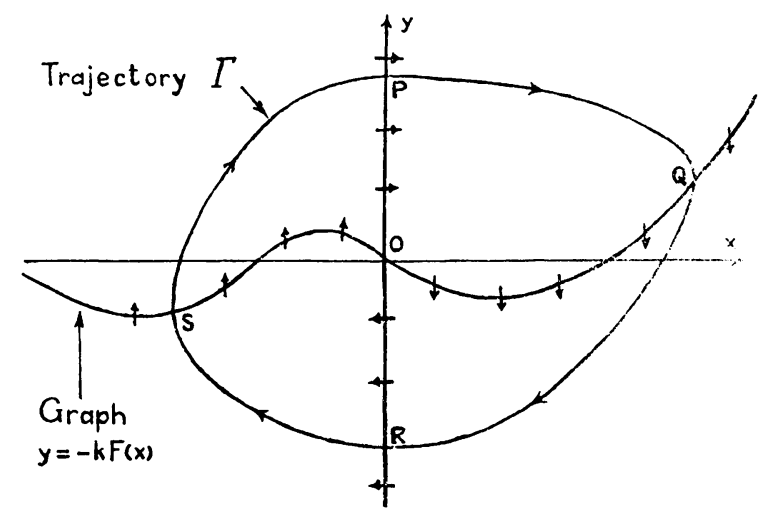

FIG. 1. 
of these arcs. By considering the total variation of $y$ and $\phi(x)$ around $\Gamma$ we obtain

$$
\begin{gathered}
\int_{\Gamma}\left|y^{\prime}\right| d t=2(p-r), \quad \int_{\Gamma}\left|\phi^{\prime}\right| d t=2[\phi(q)-\phi(s)], \\
\int_{\Gamma}\left[\left(y^{\prime}\right)^{2}+\left(\phi^{\prime}\right)^{2}\right]^{1 / 2} d t \leq 2[p-r+\phi(q)-\phi(s)],
\end{gathered}
$$

where $(0, p),(q,-k F(q)),(0, r),(s,-k F(s))$ are the coordinates of $P, Q, R, S$, respectively. Define $W(x, y) \geq 0$ by

$$
W(x, y)=\left[(y+k F(x))^{2}+2 G(x)\right]^{1 / 2} .
$$

Then (8) and (7) give $\left(x^{\prime}\right)^{2}+2 G(x)=W(x, y)^{2}$ and

$$
\left(y^{\prime}\right)^{2}+\left(\phi^{\prime}\right)^{2}=g(x)^{2}+\frac{1}{2} G(x)^{-1} g(x)^{2}\left(x^{\prime}\right)^{2}=\frac{1}{2} G(x)^{-1} g(x)^{2} W(x, y)^{2} .
$$

This and (4) give $\left(y^{\prime}\right)^{2}+\left(\phi^{\prime}\right)^{2} \geq v^{2} \omega^{2}$ on $\Gamma$, where $\omega$ is the minimum value of $W(x, y)$ on $\Gamma$. Substitution in (10) gives

$$
v \omega T \leq 2[p-r+\phi(q)-\phi(s)]=2\left[W_{P}+W_{R}+W_{\odot}+W_{s}\right],
$$

where $W_{P}, W_{Q}, W_{R}, W_{S}$ are the values of $W(x, y)$ at $P, Q, R, S$. From (11), the derivative of $\frac{1}{2} W(x, y)^{2}$ following a solution of $(8)$ is

$$
W^{\prime} W=\left(y^{\prime}+x^{\prime} k f\right)\left(y+k F^{\prime}\right)+x^{\prime} g=\left(x^{\prime}\right)^{2} k f(x) .
$$

By considering $\int W^{\prime} d t$ along the $\operatorname{arc} P Q$ we get

$$
W_{0}-W_{P}=\int_{P}^{0} W^{-1}\left(x^{\prime}\right)^{2} k f(x) d t \leq \int_{P}^{0} W^{-1}\left(x^{\prime}\right)^{2} k f_{+}(x) d t .
$$

Since $W^{-1}\left|x^{\prime}\right| \leq W^{-1}\left[\left(x^{\prime}\right)^{2}+2 G(x)\right]^{1 / 2}=1$, and $x^{\prime}>0$ on $P Q$, this gives

$$
W_{0}-W_{P} \leq k \int_{P}^{0} x^{\prime} f_{+}(x) d t=k \int_{0}^{a} f_{+}(x) d x \leq J_{1} .
$$

Integrating $W^{\prime}$ along the $\operatorname{arcs} Q R, R S, S P$, we get similarly

$$
W_{R}-W_{0} \leq J_{1}, \quad W_{S}-W_{R} \leq J_{2}, \quad W_{P}-W_{S} \leq J_{2} .
$$

Let $M$ be the point on $\Gamma$ at which $W(x, y)$ takes its minimum value $\omega$. We now discuss separately the four possible cases when $M$ lies on each of the $\operatorname{arcs} P Q, Q R, R S, S P$. In the case when $M$ lies on $P Q$ we integrate $W^{\prime}$ along the arc $M Q$ and use (13) to get

$$
W_{\odot}-\omega=\int_{M}^{Q} W^{-1}\left(x^{\prime}\right)^{2} k f(x) d t \leq k \int_{P}^{Q} x^{\prime} f_{+}(x) d t \leq J_{1} .
$$

It then follows from (15), (16) that

$$
\begin{aligned}
7 J_{1}+3 J_{2} & \geq 4\left(W_{0}-\omega\right)+3\left(W_{R}-W_{\odot}\right)+2\left(W_{S}-W_{R}\right)+\left(W_{P}-W_{S}\right), \\
& =W_{P}+W_{0}+W_{R}+W_{S}-4 \omega .
\end{aligned}
$$

In the case when $M$ lies on $Q R$ we integrate $W^{\prime}$ along the arc $M R$ to get $W_{R}-\omega \leq J_{1}$ instead of (16). Then (14), (15) give

$$
\begin{aligned}
5\left(J_{1}+J_{2}\right) & \geq 4\left(W_{R}-\omega\right)+3\left(W_{S}-W_{R}\right)+2\left(W_{P}-W_{S}\right)+\left(W_{Q}-W_{P}\right), \\
& =W_{P}+W_{Q}+W_{R}+W_{S}-4 \omega .
\end{aligned}
$$


In the case when $M$ lies on $R S$ we integrate $W^{\prime}$ along the arc $M S$ to get $W_{s}-\omega \leq J_{2}$ instead of (16). Then (14), (15) give

$$
\begin{aligned}
3 J_{1}+7 J_{2} & \geq 4\left(W_{S}-\omega\right)+3\left(W_{P}-W_{S}\right)+2\left(W_{\odot}-W_{P}\right)+\left(W_{R}-W_{\odot}\right), \\
& =W_{P}+W_{Q}+W_{R}+W_{S}-4 \omega .
\end{aligned}
$$

In the case when $M$ lies on $S P$ we integrate $W^{\prime}$ along the $\operatorname{arc} M P$ to get $W_{P}-\omega \leq J_{2}$ instead of (16). Then (14), (15) give

$$
5\left(J_{1}+J_{2}\right) \geq 4\left(W_{P}-\omega\right)+3\left(W_{0}-W_{P}\right)+2\left(W_{R}-W_{0}\right)+\left(W_{S}-W_{R}\right),
$$

which is (18) again. Each of the three possibilities (17), (18), (19) gives

$$
W_{P}+W_{Q}+W_{R}+W_{S} \leq 4 \omega+3\left(J_{1}+J_{2}\right)+4 \max \left(J_{1}, J_{2}\right) .
$$

This and (12) give

$$
T \leq v^{-1}\left[8+6 \omega^{-1}\left(J_{1}+J_{2}\right)+8 \omega^{-1} \max \left(J_{1}, J_{2}\right)\right] .
$$

If $(m, \mu)$ are the coordinates of the point $M$ on $\Gamma$ at which $W(x, y)$ attains its minimum value $\omega$ then $f(m)=0$ by (13). Hence $m$ lies outside $(-b, \beta)$ by (5) and therefore $G(m) \geq$ $\min \{G(-b), G(\beta)\}=\frac{1}{2} \gamma$. From (11),

$$
\omega^{2}=(\mu+k F(m))^{2}+2 G(m) \geq 2 G(m) \geq \gamma .
$$

Replace $\omega^{-1}$ by $\gamma^{-1 / 2}$ in (21) to obtain (6). This establishes Theorem 1.

\section{Simple improvements.}

Theorem 2. If $f(x), g(x)$ satisfy (2), (4), (5) and

$$
\operatorname{sign} f(x)=\operatorname{sign}[(x+b)(\beta-x)]
$$

for all $x$, then (6) can be replaced by

$$
T \leq v^{-1}\left\{8+10 \gamma^{-1 / 2}\left(J_{1}+J_{2}\right)\right\} .
$$

Proof. Since the abscissa $m$ of $M$ satisfies $f(m)=0$, (23) shows that $m$ is either $-b$ or $\beta$ and that $f(x)>0$ for all $x$ between $m$ and 0 . It follows that $M$ cannot lie on the arc $P Q$ because otherwise $W(x, y)$ would be strictly increasing along the arc $P M$ by (13) and $W(x, y)$ would not take its minimum value at $M$. For the same reason $M$ cannot lie on $R S$. The possibilities (17), (19) are therefore excluded and (20) can be replaced by (18). This leads to (24). When $J_{1} \neq J_{2},(24)$ is a sharper inequality than (6).

Theorem 3. If $f(x), g(x)$ satisfy (2), (4), (5) and

$$
f(-x)=f(x), \quad g(-x)=-g(x),
$$

for all $x$, then (6) can be replaced by

$$
T \leq v^{-1}\left\{8+6 \gamma^{-1 / 2}\left(J_{1}+J_{2}\right)\right\} .
$$

Proof. From (3) and (25) we get $F(x)=-F(x), G(-x)=G(x)$. If $x(t), y(t)$ is a solution of (8) then so is $-x(t),-y(t)$ and as the point $(x(t), y(t))$ travels around $\Gamma$, the point $(-x(t),-y(t))$ travels the curve $\Gamma^{*}$ got by reflecting $\Gamma$ in the origin. Since $\Gamma, \Gamma^{*}$ are trajectories of (8) which intersect they must coincide. That is, $\Gamma$ is symmetric with respect to the origin. If $M^{*}$ is the reflection of a point $M$ at which $W(x, y)$ attains its 
minimum value $\omega$ on $\Gamma$ then $W(x, y)=\omega$ at $M^{*}$ also because $W(-x,-y)=W(x, y)$ by (11). We can therefore assume that $M$ lies either on $S P$ or on $P Q$. If $M$ lies on $S P$ then $M^{*}$ lies on $Q R$ and we get both inequalities $W_{P}-\omega \leq J_{2}, W_{R}-\omega \leq J_{1}$ instead of (16). Then (14) and (15) give

$$
\begin{aligned}
3\left(J_{1}+J_{2}\right) & \geq 2\left(W_{P}-\omega\right)+2\left(W_{R}-\omega\right)+\left(W_{\odot}-W_{P}\right)+\left(W_{S}-W_{R}\right), \\
& =W_{P}+W_{Q}+W_{R}+W_{S}-4 \omega .
\end{aligned}
$$

If $M$ lies on $P Q$ then $M^{*}$ lies on $R S$ and we get both $W_{Q}-\omega \leq J_{1}$ and $W_{S}-\omega \leq J_{2}$. Then (15) gives

$$
3\left(J_{1}+J_{2}\right) \geq 2\left(W_{Q}-\omega\right)+2\left(W_{S}-\omega\right)+\left(W_{R}-W_{o}\right)+\left(W_{P}-W_{S}\right)
$$

which reduces to (27) again. It follows that (20) can be replaced by (27) and the argument then leads to (26). For van der Pol's equation (26) gives $T \leq 8(1+k)$ which overestimates $T$ by a factor of 5 when $k$ is large.

4. Other improvements. So far information about the rate of growth of the damping function $F(x)$ has not been used. This will now be taken into account.

Theorem 4. Suppose that $f(x), g(x)$ satisfy (2), (4), (5) and that $c, d$ are positive constants such that

$$
2+G(x)^{-1} k^{2} F(x)^{2} \geq 2 c^{2}\left\{1+2 \gamma^{-1 / 2}\left(J_{1}+J_{2}\right)\right\}^{2}
$$

for all $x$ with $2 G(x)>d$. Then (6) can be replaced by

$$
\frac{1}{4} v T \leq 1+\gamma^{-1 / 2}\left[J_{1}+J_{2}+\max \left(J_{1}, J_{2}\right)\right]+\max \left(c^{-1},(d / \gamma)^{1 / 2}\right) .
$$

Proof. Let $N$ be the point on $\Gamma$ at which $W(x, y)$ takes its maximum value $\Omega$ on $\Gamma$. By considering $\int W^{\prime} d t$ along the arc $M N$ and substituting for $W^{\prime}$ from (13) we get

$$
\Omega-\omega=\int_{M}^{N} W^{-1}\left(x^{\prime}\right)^{2} k f(x) d t \leq \int_{\Gamma}\left|x^{\prime}\right| k f_{+}(x) d t \leq 2\left(J_{1}+J_{2}\right) .
$$

This and (22) give

$$
\Omega \leq \omega+2\left(J_{1}+J_{2}\right) \leq \omega\left\{1+2 \gamma^{-1 / 2}\left(J_{1}+J_{2}\right)\right\} .
$$

The derivative of $L(x, y)=y^{2}+2 G(x)$ following a solution of (8) is

$$
L^{\prime}=2 y y^{\prime}+2 g(x) x^{\prime}=2 k g(x) F(x) .
$$

The maximum value of $L(x, y)$ on $\Gamma$ is therefore attained at a point where $F(x)=0$. But (11) shows that $L(x, y)=W(x, y)^{2}$ when $F(x)=0$. Hence $L(x, y) \leq \Omega^{2}$ at all points of $\Gamma$. In particular, this holds at the point $Q=(q,-F(q))$ at which $W_{o}^{2}=2 G(q)$. Therefore

$$
\Omega^{2} \geq L(q,-F(q))=\left[1+\frac{1}{2} G(q)^{-1} k^{2} F(q)^{2}\right] W_{0}^{2} .
$$

Either $d<2 G(q)$ or $d \geq 2 G(q)=W_{0}^{2}$. In the former case (28) holds for $x=q$ and (31) gives $\Omega \geq c\left[1+2 \gamma^{-1 / 2}\left(J_{1}+J_{2}\right)\right] W_{Q}$. Then (30) gives $\omega \geq c W_{Q}$. Hence, $W_{Q} \leq \max$ $\left(d^{1 / 2}, \omega / c\right)$ in both cases. Since $W_{s}=2 G(s)$, the same argument can be applied to the point $S$, instead of $Q$, to give the same bound for $W_{S}$ and therefore

$$
W_{o}+W_{S} \leq 2 \max \left(d^{1 / 2}, \omega / c\right) \text {. }
$$


As in Sec. 2, we now discuss separately the four possible cases when $M$ lies on each of the $\operatorname{arcs} P Q, Q R, R S, S P$. When $M \in P Q,(16)$ holds and then (15) gives

$$
4 J_{1}+2 J_{2} \geq 2\left(W_{Q}-\omega\right)+2\left(W_{R}-W_{Q}\right)+\left(W_{P}-W_{S}\right)+\left(W_{S}-W_{R}\right) .
$$

When $M \in Q R$ we have $W_{R}-\omega \leq J_{1}$. Then (15) gives

$$
2\left(J_{1}+J_{2}\right) \geq 2\left(W_{R}-\omega\right)+\left(W_{S}-W_{R}\right)+\left(W_{P}-W_{S}\right) .
$$

When $M \in R S$ we have $W_{S}-\omega \leq J_{2}$. Then (14) and (15) give

$$
2 J_{1}+4 J_{2} \geq 2\left(W_{S}-\omega\right)+2\left(W_{P}-W_{S}\right)+\left(W_{0}-W_{P}\right)+\left(W_{R}-W_{0}\right) \text {. }
$$

When $M \in S P$ we have $W_{P}-\omega \leq J_{2}$. Then (14) and (15) give

$$
2\left(J_{1}+J_{2}\right) \geq 2\left(W_{P}-\omega\right)+\left(W_{\odot}-W_{P}\right)+\left(W_{R}-W_{\odot}\right) .
$$

In all four cases these inequalities give

$$
W_{P}+W_{R} \leq 2 \omega+2\left(J_{1}+J_{2}\right)+2 \max \left(J_{1}, J_{2}\right) .
$$

Substituting (32) and (33) in (12) we get

$$
\frac{1}{4} v T \leq 1+\omega^{-1}\left[J_{1}+J_{2}+\max \left(J_{1}, J_{2}\right)\right]+\max \left(c^{-1}, d^{1 / 2} \omega^{-1}\right) .
$$

Using (22), we can replace $\omega^{-1}$ by $\gamma^{-1 / 2}$ to obtain (29).

Theorem 5. If both (23) and (25) are added to the hypotheses of Theorem 4 then (29) can be replaced by

$$
\frac{1}{4} v T \leq 1+\frac{1}{2} \gamma^{-1 / 2}\left(J_{1}+J_{2}\right)+\max \left(c^{-1},(d / \gamma)^{1 / 2}\right) .
$$

Proof. As in the proof of Theorem 3, (25) implies that the minimum value $\omega$ of $W(x, y)$ is taken at two points $M, M^{*}$ on $\Gamma$ which are symmetrically arranged with respect to the origin. As in the proof of Theorem 2, (23) implies that $M, M^{*}$ do not lie on the $\operatorname{arcs} P Q$ and $R S$. It can therefore be assumed that $M \in S P, M^{*} \in Q R$. As in Sec. 2, this implies both $W_{P}-\omega \leq J_{2}$ and $W_{R}-\omega \leq J_{1}$. Hence (33) can be replaced by the sharper inequality $W_{P}+W_{R} \leq 2 \omega+J_{1}+J_{2}$. Substituting this and (32) in (12) we obtain (34).

5. Comments. For the case of van der Pol's equation, $F(x)=x-\frac{1}{3} x^{3}, G(x)=\frac{1}{2} x^{2}$ and the left-hand side of (28) is monotonic for $x^{2}>3$. In this case (28) is satisfied by taking $d>3$ and $c^{2}=(3+8 k)^{-2}\left[9+k^{2}(d-3)^{2}\right]$. Then (34) gives

$$
T \leq 4+(8 k / 3)+4 \max \left\{d^{1 / 2},(3+8 k)\left[9+k^{2}(d-3)^{2}\right]^{-1 / 2}\right\} .
$$

The arbitrary constant $d$ can be chosen in the range $3<d<\infty$ so as to minimize the right-hand side. With $d=7$ it gives $T \leq 4(1+\sqrt{7})+(8 k / 3)$ for all $k>0$. For large $k$ this is much closer to Liénard's asymptotic formula $T \sim 1.614 k$ than is the inequality $T \leq 8(1+k)$ obtained from (26).

A general method for finding period bounds for $2 \times 2$ autonomous systems was given by Diliberto [2] and modified by Lau [4]. The basic idea of their method is to enclose the trajectory $\Gamma$ within a suitable region of known area and then to estimate its length by finding its maximum curvature. For the system (8) it is a difficult task to locate $\Gamma$ with enough precision to use their method effectively. Their basic idea is replaced in the present paper by the inequality (10). One of the advantages of this method is that it can avoid the task of locating $\Gamma$. 


\section{RefERENCES}

1. T. W. Davies and E. M. James, Nonlinear differential equations, Addison-Wesley, Reading, Mass., 1966

2. S. P. Diliberto, Bounds for periods of periodic solutions. In Contributions to the theory of nonlinear oscillations (ed. S. Lefschetz), Vol. III, Princeton Univ. Press, Princeton, N. J., 1956, pp. 269-275

3. D. Graffi, Sopra alcune equazioni differenziali della radiotechnica, Mem. Accad. Sci. Ist. Bologna Cl. Sci. Fis. (9) 9, 145-153 (1942)

4. P. J. Lau, Bounds for the lengths and periods of closed orbits of two-dimensional autonomous systems of differential equations, J. Differential Equations 3, 330-342 (1967)

5. P. J. Ponzo and N. Wax, On certain relaxation oscillations asymptotic solutions, J. Soc. Indust. Appl. Math. 13, 740-766 (1965)

6. G. Sansone and R. Conti, Nonlinear differential equations, Pergamon Press, Oxford, 1964

7. M. Urabe, Nonlinear autonomous oscillations, Academic Press, New York, 1967 